\title{
Experimental Investigation of Heat Transfer in Stagnant Bed for Processing of Agriculture Products
}

\author{
Jan Havlík, Jan Opatřil \\ CTU in Prague, Faculty of Mechanical Engineering \\ Technická 4, 16607 Praha, Czechia \\ Jan.Havlik@fs.cvut.cz; Jan.Opatril@fs.cvut.cz
}

\begin{abstract}
The paper deals with the heat transfer associated with processing of agriculture products. Granular materials such as legumes or cereals often require preheating to certain temperature for its processing. This can be secured by heat exchanger, where it is necessary to have a knowledge about heated material behaviour for technically and economically proper design. Commonly used construction type of heat exchangers for the food processing is a platen heat exchanger. Thus, the heat transfer coefficients between the planar heated surface and the stagnant bed are main subject of this paper. Heat transfer is theoretically described based on the penetration model that considers the bed of particles as one continuous phase. For experimental investigation of heat transfer, a stand for heating a packed bed of material was designed and manufactured. Selected materials were experimentally measured at different levels of heating temperature to gain the time dependence of the heat transfer coefficients and the temperature distribution in the bed of material. The temperature in the bed of material rapidly increases at area close to the heating surface, while at a greater distance a temperature increase is very small even after $30 \mathrm{~min}$. The temperature increases faster in material with the lower specific heat capacity. The heat transfer coefficient is not affected by heating temperature but only by the change of material properties. In agreement with both experimental and theoretical results, the heat transfer coefficient decreases significantly at the time of heating. The most intense heat transfer occurs in the first heating phase (in the order of a few minutes), after which the heat transfer is stabilized and lowered. These results may be used for designing the mentioned application of heat exchangers.
\end{abstract}

Keywords: Heat transfer, Stagnant bed, Temperature distribution, Agriculture products.

\section{Introduction}

In many industrial applications, it is necessary to pre-heat the agriculture products, such as cereals, legumes, etc., to a certain temperature before further processing. Steam or water-heated heat exchangers are often used for preheating these materials. The accurate prediction of heat transfer behaviour is of great importance for the design and optimizing the operation of these facilities. Modelling of heat transfer is based on a model that considers the bed of particles as one continuous phase. This is usually called the penetration model [1,2]. Not only the heat transfer but also the temperature distribution in the bed of heated material is important, in order to maintain the basic requirement which is the maximum allowable material temperature. The heating temperature is limited by prevention the nutrients degradation and on the other side influence required heat transfer surface, which impacts to the costs.

The aim of the article is to describe the heat transfer for legumes and similar products in agriculture applications. The properties (the bulk density, the particle density, the specific heat capacity) of plant materials vary very often, depending on the place of origin, weather, harvest conditions, storage, etc., therefore the experiments with soya beans and two types of lightweight ceramic aggregate (LWA) were carried out to evaluate the influence of the change in thermal properties. LWA was chosen due to similar basic thermal properties, which are differentiated by the way of preparation and are so well described.

\section{Theoretical Approach to Heat transfer in the Stagnant Bed}

The heat transfer from a hot surface to free flowing and mechanically agitated particulate dry material and the subsequent problem of heat transfer to stirred beds during contact drying has been investigated by Schlünder and co-workers $[3,4]$. In both cases the so-called penetration model was applied [4]. 


\subsection{Heat transfer from the heated wall to the packed bed}

Application of the penetration model is well described in [2] and [5]. In Fig. 1, the scheme of heat transfer from the heated wall to the particulate material is shown. There is a steep temperature drop in the immediate vicinity of the wall, $\vartheta_{w}-\vartheta_{0}$, followed by a further decrease toward the interior of the bed, $\vartheta_{w}-\vartheta_{0}$ (average caloric bed temperature).

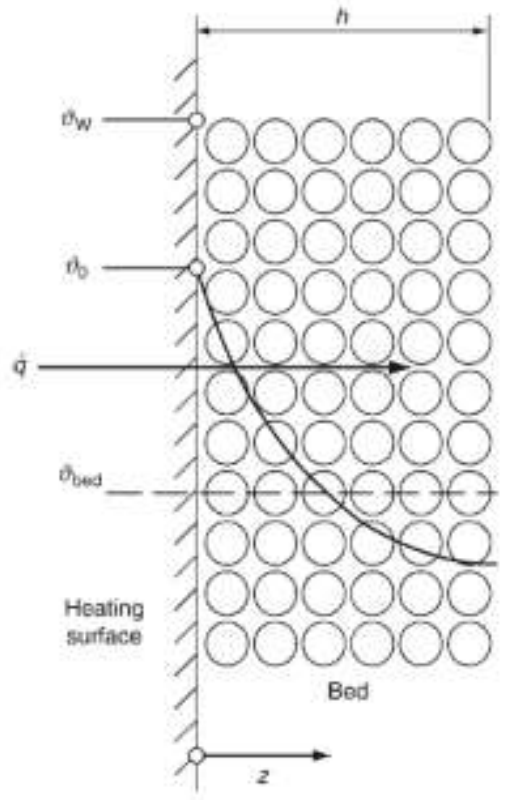

Fig. 1: Scheme of heat transfer from a wall to an adjoining bed of particles [2].

The temperature jump $\vartheta_{w}-\vartheta_{0}$, and the temperature difference $\vartheta_{0}-\vartheta_{\text {bed }}$ are assigned to contact heat resistance, $1 / \alpha_{w s}$, and to penetration resistance, $1 / \alpha_{b e d}$, respectively. Therefore, it is

$$
\begin{gathered}
\alpha_{\mathrm{ws}}=\dot{\mathrm{q}} /\left(\vartheta_{\mathrm{w}}-\vartheta_{0}\right) \\
\alpha_{\text {bed }}=\dot{\mathrm{q}} /\left(\vartheta_{0}-\vartheta_{\text {bed }}\right)
\end{gathered}
$$

The overall heat transfer coefficient (HTC) $\alpha$ is

and

$$
\alpha=\dot{\mathrm{q}} /\left(\vartheta_{\mathrm{w}}-\vartheta_{\mathrm{bed}}\right)
$$

$$
\frac{1}{\alpha}=\frac{1}{\alpha_{w s}}+\frac{1}{\alpha_{\text {bed }}}
$$

Using the standard boundary condition of constant temperature $\vartheta_{0}$, the time-averaged heat penetration coefficient of the stagnant bed $\alpha_{\text {bed }}$ is obtained to

$$
\alpha_{\text {bed }}=\frac{2}{\sqrt{\pi}} \frac{\sqrt{(\rho \cdot c \cdot \lambda)_{\text {bed }}}}{\sqrt{t_{R}}}
$$


Here, $\rho, c, \lambda$ are respectively the density, heat capacity at constant pressure and the thermal conductivity, $t_{R}$ is the residence time of the bed on the heating surface. The contact HTC between the wall and the bed $\alpha_{W S}$ is obtained to

$$
\alpha_{\mathrm{WS}}=\varphi \alpha_{\mathrm{WP}}+\alpha_{\mathrm{rad}}
$$

where $\varphi$ is the surface coverage factor, $\alpha_{W P}$ is the wall-particle HTC, $\alpha_{\text {rad }}$ is the HTC by radiation.

$$
\alpha_{\mathrm{WP}}=\frac{4 \lambda_{\mathrm{g}}}{\mathrm{d}}\left[\left(1+\frac{2(\mathrm{l}+\mathrm{d})}{\mathrm{d}}\right) \ln \left(1+\frac{\mathrm{d}}{2(\mathrm{l}+\delta)}\right)-1\right]
$$

where $l$ is the modified mean free path of gas molecules, $\lambda_{g}$ is the thermal conductivity of the gas, $d$ is the particle diameter, $\delta$ is the surface roughness of the particles.

$$
\alpha_{\text {rad }}=4 C_{W, \text { bed }} \mathrm{T}^{3}
$$

where $T$ is the absolute temperature and the overall radiation exchange coefficient $C_{W, b e d}$ is calculated as

$$
\mathrm{C}_{\mathrm{W}, \mathrm{cbed}}=\sigma /\left(1 / \varepsilon_{\mathrm{W}}+1 / \varepsilon_{\mathrm{bed}}-1\right)
$$

where $\varepsilon_{W}, \varepsilon_{\text {bed }}$ are the emission coefficients of wall and bed, respectively. The description of calculation and the determination of the parameters above are described in detail in [2].

\section{Experimental Set-up}

\subsection{Experimental stand}

The measuring stand consists of a heated chamber made of thick insulation mounted on the supporting frame, see Fig. 2. The basic external dimensions of the structure are $1000 \times 840 \times 590 \mathrm{~mm}(\mathrm{H} \mathrm{x} \mathrm{W} \mathrm{x} \mathrm{D).} \mathrm{The} \mathrm{chamber} \mathrm{has} \mathrm{square} \mathrm{cross-}$ section with dimensions $222 \times 222 \mathrm{~mm}$ with adjustable length. The heating of the material is carried out by means of two electric heating elements, which are mounted on an aluminium plate approximately $10 \mathrm{~mm}$ thick to ensure uniform heat flow into the heated material. The power control of heating elements is ensured by the PID controller with a surface thermocouple attached to the surface of the plate, which is in contact with the heated material. 


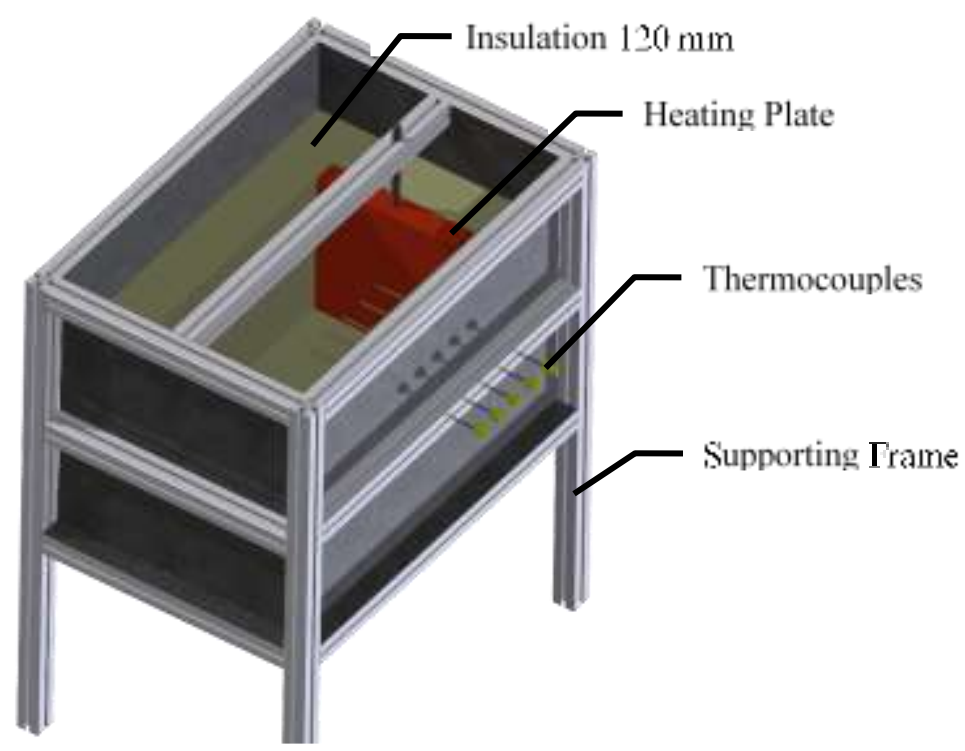

Fig. 2: Experimental stand drawing.

Measurement of total power is secured by an electricity meter with pulse output, so that it is possible to monitor the actual consumption for heating in time. Temperature profile measurement is done by 3 thermocouples immersed in the bed, and there are mounted 4 surface thermocouples for controlling the heating temperature and for evaluation of thermal loses to the surroundings. Thermocouples and pulse output are connected to the data logger. A view of the device during measurement is shown in Fig. 3, where the heating plate and the chamber equipped with thermocouples for temperature profile measurement are visible.

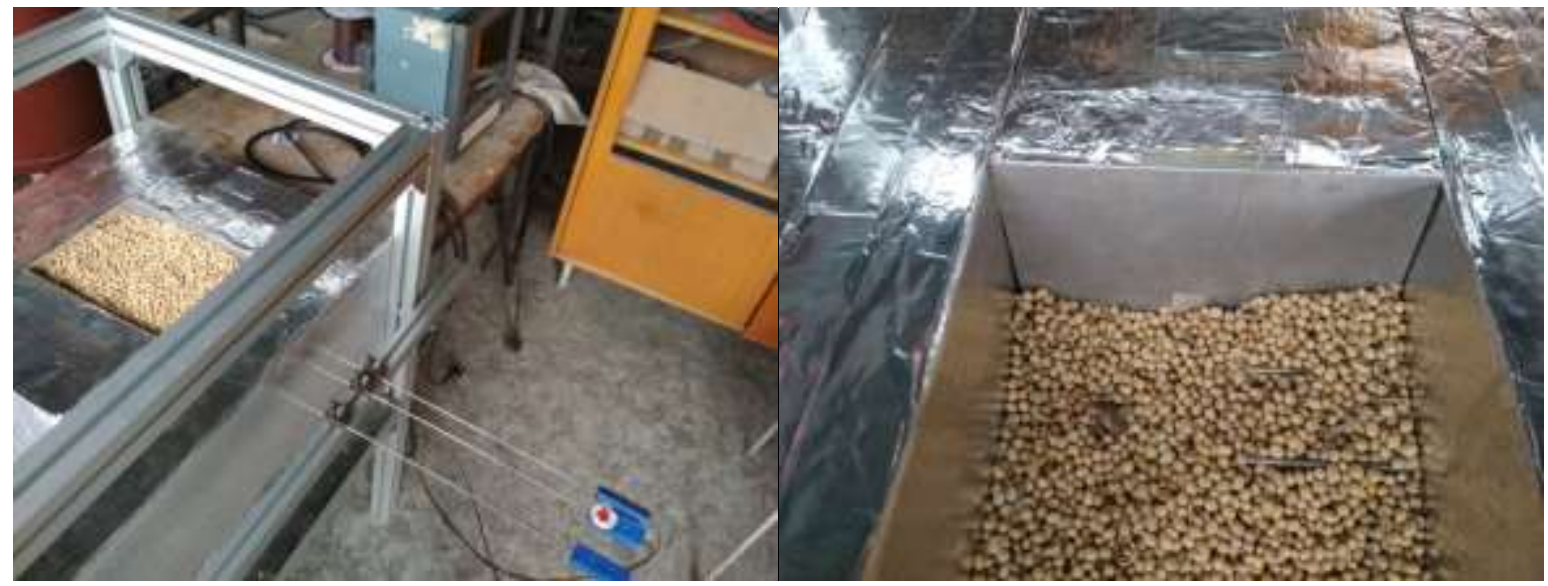

Fig. 3: Experimental stand.

Measurement parameters, i.e. the number of thermocouples, their distance and frequency of data collection, can be adjusted to approach as close as possible the real parameters of industrial process (in this case extrusion line operation).

\subsection{Experimental Procedure}

The unit with empty chamber is heated to the desired heating temperature before starting the experiment to eliminate heat loses by heat accumulation in solids, then the investigated material is placed into the chamber while all the monitored parameters are recorded. Time dependence of material heating in certain distances from the heating surface is determined by measurement of temperature course in these points. The dependence of the heat transfer coefficient on 
the heating time is evaluated from the course of the heating performance over time, the knowledge of temperatures and the size of the heated surface.

The material temperatures are measured at three distances from the heating plate, i.e. $10 \mathrm{~mm}, 25 \mathrm{~mm}$ and $60 \mathrm{~mm}$, and the heating temperature is measured by surface thermocouple directly on the heating plate surface. The chamber length was fixed to $120 \mathrm{~mm}$, thus the dimensions of heated space are $222 \times 222 \times 120 \mathrm{~mm}$.

The experiments were carried out in the range of surface temperatures from $90{ }^{\circ} \mathrm{C}$ to $130{ }^{\circ} \mathrm{C}$.

\subsection{Properties of Investigated Materials}

For investigation, soybeans and two different lightweight ceramic aggregates were selected, which simulates different conditions of heated material. Properties of select materials are listed in Table 1. The reason for selecting LWA is described above.

Table 2: Properties of Investigated Materials.

\begin{tabular}{|l|c|c|c|}
\hline & Soybeans & LWA - 02 & LWA - 48 \\
\hline Mean particle diameter $[\mathrm{mm}]$ & 6 to 8 & 0 to 2 & 4 to 8 \\
\hline Bulk density $\left[\mathrm{kg} / \mathrm{m}^{3}\right]$ & 700 & 575 & 350 \\
\hline Particle density $\left[\mathrm{kg} / \mathrm{m}^{3}\right]$ & 1200 & 1050 & 700 \\
\hline Thermal conductivity $[\mathrm{W} / \mathrm{mK}]$ & 0.123 & 0.12 & 0.10 \\
\hline Specific heat capacity $[\mathrm{kJ} / \mathrm{kgK}]$ & 2.03 & 0.90 & 0.90 \\
\hline
\end{tabular}

\section{Results}

The time dependence of the temperature distribution in the bed of material is shown in Fig. 4. The graphs show the temperature distribution in the bed in various distances from the heated surface $(10,25$ resp. $60 \mathrm{~mm})$. For temperature profiles, measurements are distinguished by color and distance of thermocouples by line type. The red and blue lines represent the comparison of two different heating temperatures. The distance $0 \mathrm{~mm}$ represents the temperature on the heated plate surface.

Initial temperature drop is given by preheating the heated chamber to the desired temperature and then dropping the temperature in the chamber after filling with material. 

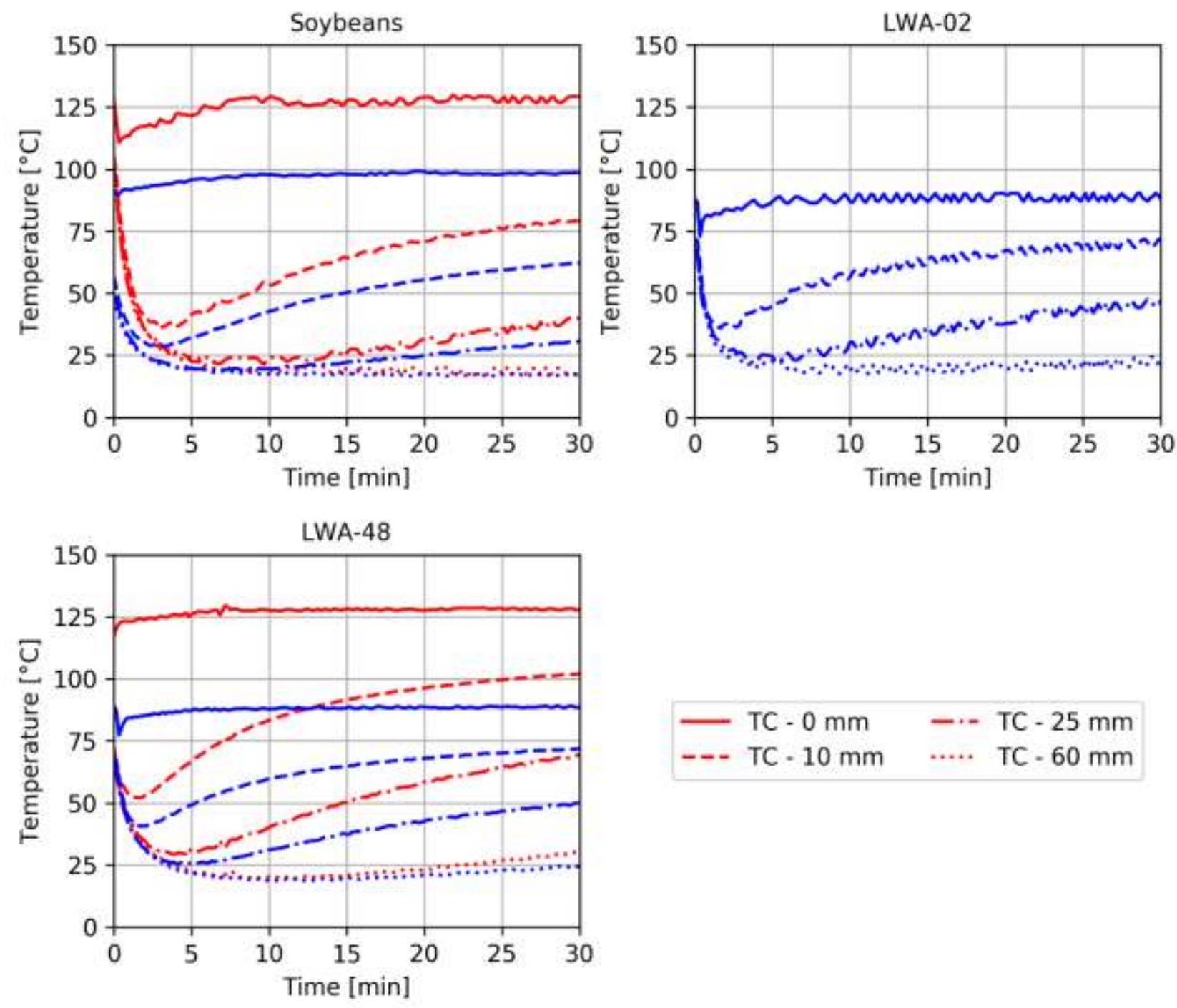

Fig. 4: Temperature profiles.

The temperature rapidly increases at area close to the heating surface (distance $10 \mathrm{~mm}$ ) while in the distance of 60 $\mathrm{mm}$ a temperature increase is very small even after $30 \mathrm{~min}$. This applies to all heating temperature ranges from 90 to $130^{\circ} \mathrm{C}$. The temperature increases faster in material with the lower specific heat capacity (LWA).

The time dependence of the heat transfer coefficients (HTC) is shown in Fig. 5. Experimentally determined values of the HTC are compared with the theoretical values calculated according to the penetration model described in the part 2.1. 


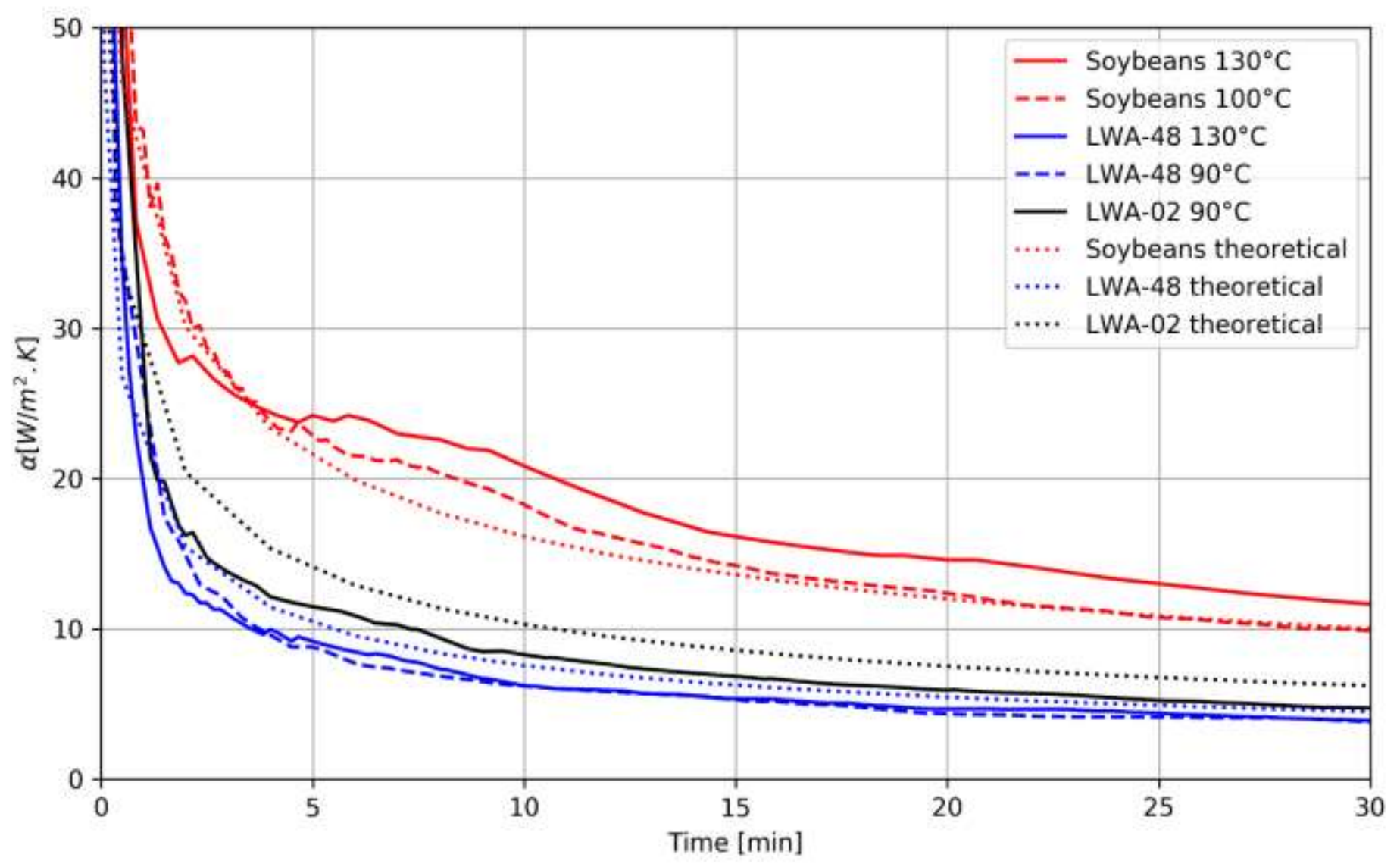

Fig. 5: Time dependence of the heat transfer coefficient.

From the results for LWA, it is evident that the HTC is not affected by heating temperature but only by the change of material properties. The difference between the HTC for soybeans can be caused by the fact that at higher heating temperatures may occur to partially evaporate water from plant material in areas close to heating plate, which may have affected an increase in the HTC. The higher values of the HTC for soybeans are mainly caused by the higher specific heat capacity of soybeans in comparison with LWA. When compare two types of LWA, it is evident that higher density increases the HTC.

In agreement with both experimental and theoretical results, the HTC decreases significantly at the time of heating. The experimental results are slightly lower than the theoretical. The most intense heat transfer occurs in the first heating phase (in the order of a few minutes), after which the heat transfer is stabilized and lowered.

Investigated materials (generally as well as agricultural products) can have inhomogeneous physical properties. A deviation of $20 \%$ when determining the density, the thermal conductivity or the specific heat capacity results in a change of the HTC about roughly $10 \%$. Other properties such as sphericity, particle size, etc., have a small impact on the HTC.

\section{Conclusions}

Heat transfer between the planar heated surface and the stagnant bed of soybeans and LWA was experimentally investigated. A stand for heating a packed bed of material was designed and manufactured. For the theoretical description of heat transfer between the wall and the bed of particles the penetration model is used.

The temperature rapidly increases at area close to the heating surface (distance $10 \mathrm{~mm}$ ), while at a greater distance a temperature increase is very small even after 30min. This applies to all heating temperature ranges from 90 to $130^{\circ} \mathrm{C}$. The temperature increases faster in material with the lower specific heat capacity (LWA).

The HTC is not affected by heating temperature but only by the change of material properties. Materials with a higher value of the higher specific heat capacity or the density have higher values of the HTC. In agreement with both experimental 
and theoretical results, the HTC decreases significantly at the time of heating. The experimental results are slightly lower than the theoretical. The heat transfer is the most intensive in the first heating phase (in the order of a few minutes), after which the heat transfer is stabilized and lowered. The evaluation of the HTC is very sensitive on accurate determining the density, the thermal conductivity and the specific heat capacity. These results may be used for designing the mentioned application of heat exchangers.

\section{Acknowledgements}

This work has been supported by the Technology Agency of the Czech Republic, project no. TJ01000192.

\section{References}

[1] R. Zhang, H. Yang, J. Lu and Y. Wual, "Theoretical and experimental analysis of bed-to-wall heat transfer in heat recovery processing," Powder Technology, vol. 249, pp. 186-195, 2013.

[2] VDI heat atlas. New York: Springer, 2010.

[3] E. U. Schlünder and N. Mollekopf, "Vacuum Contact Drying of Free Flowing Mechanically Agitated Particulate Material," Chem. Eng. Process, vol. 18, pp. 93-111, 1983.

[4] E. Tsotsas, E.U. Schlünder, "Contact Drying of Mechanically Agitated Particulate Material in the Presence of Inert Gas," Chem. Eng. Process, vol. 20, pp. 277-285, 1986.

[5] A. S. Mujumdar, Handbook of Industrial Drying. New York: Marcel Dekker, 1990. 